\title{
Poisson-Boltzmann calculations of ions in charged capillaries
}

\author{
Wai Yin Lo and Kwong-Yu Chan \\ Department of Chemistry, The University of Hong Kong, Hong Kong
}

(Received 19 January 1994; accepted 4 April 1994)

\begin{abstract}
The Poisson-Boltzmann (PB) equation of ions inside an infinitely long charged pore is solved using two different boundary conditions of the electric field due to the pore wall. The results show that the boundary condition generally adopted in the literature leads to a violation of electroneutrality in the ion-pore system.
\end{abstract}

\section{INTRODUCTION}

The behavior of electrolytes in capillaries is important for biological and industrial membranes, nerve channels, and porous electrodes. It is desirable to relate transport and thermodynamic properties of such systems to their molecular and electrostatic parameters. Some theoretical and simulation works have been reported for electrolytes inside cylindrical pores. The nonlinear Poisson-Boltzmann (PB) equation for the system with point ions was solved approximately by Levine et al. ${ }^{1}$ and Olivares et al. ${ }^{2}$ It has also been solved numerically by Signal and Ginsburg, ${ }^{3}$ and more recently by Vlachy and co-workers. ${ }^{4-7}$ The hypernetted chain theory/ mean spherical approximation theory (HNC/MSA) has been solved by Lozada-Cassou and co-workers ${ }^{8,9}$ and Vlachy and McQuarrie $^{10}$ for ions around a charged cylinder. For ions inside a charged capillary, the HNC/MSA was solved by Vlachy and McQuarrie ${ }^{11}$ and recently by Yeomans et al. ${ }^{12}$ Monte Carlo simulations have also been performed. ${ }^{4-7,11}$ The theories compare well with simulation data except in some cases of 2:2 electrolyte. ${ }^{5}$ Because of its simplicity, the PB equation is an attractive theory and is convenient for testing simulation results. An infinitely long hollow cylinder is usually assumed to be the boundary within which the ions move. For the comparison with simulation results of charged hard spheres, the PB model is usually modified so that while the ions are point ions inside the pore, they have a closest approach to the wall equal to the hard sphere radius. ${ }^{4-7,11}$ It is commonly assumed that the electric field due to the wall at the closest approach of the ions is the same as that at the wall. Unfortunately, while this is a convenient assumption, it leads to the violation of the overall electroneutrality of the system.

In this paper, the Poisson-Boltzmann equation was solved using the conventional boundary condition of electric field due to the wall. The violation of electroneutrality is demonstrated by integrating the resulting density profiles of the ions inside the pore. A simple modification of the boundary condition, however, is shown to give overall electroneutrality.

\section{POISSON-BOLTZMANN EQUATION}

The model investigated here is generally used in the literature ${ }^{4-7.11}$ and is shown in Fig. 1. The location of charges of the wall is separated from the physical wall with a distance of an ionic radius. In effect, this model assumes point ions inside the pore but the ions cannot approach the wall closer than an ionic radius. Without loss of generality, the wall is chosen to be negatively charged with a surface charge density of $\sigma_{s}$. For simplicity, we shall consider a symmetric electrolyte with only two ion species. The PoissonBoltzmann equation in the cylindrical pore is

$$
\frac{1}{r} \frac{d}{d r}\left[r \frac{d \psi(r)}{d r}\right]=-\frac{4 \pi \rho_{e}(r)}{\epsilon_{0} \epsilon_{r}}
$$

and the charge density is defined by

$$
\begin{aligned}
\rho_{e}(r)= & z_{+} e n_{+0} \exp \left[-\beta z_{+} e \psi(r)\right] \\
& +z_{-} e n_{-0} \exp \left[-\beta z_{-} e \psi(r)\right] .
\end{aligned}
$$

Here, $\psi(r)$ is the electrostatic potential at a distance $r$ from the center, $n_{+0}, n_{-0}$ are the number densities of cations and anions in the absence of an external field, $e$ is the electrostatic charge, $z_{+}$and $z_{-}$are the charge valences of the cations and anions, $\beta$ is the Boltzmann factor $1 / k T, k$ is the Boltzmann constant, $T$ is the absolute temperature, $\epsilon_{0}$ is the permittivity of free space, and $\epsilon_{r}$ is the relativity permittivity of the medium.

The solution of the above differential equation is subject to boundary conditions at the center of the pore and at the pore wall. Due to the cylindrical symmetry, the potential gradient at the center of the pore vanishes so

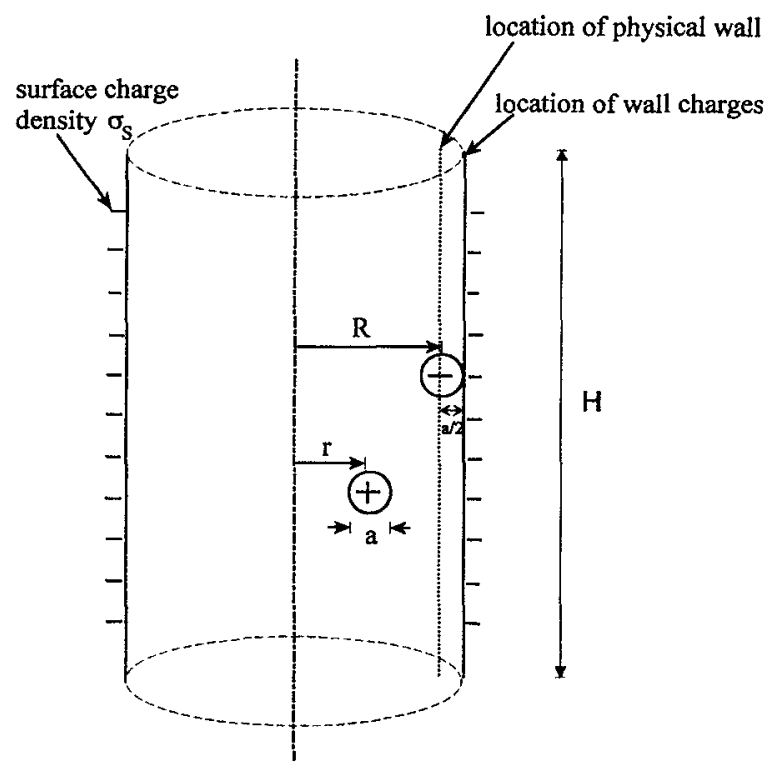

FIG. 1. A charged cylindrical pore with ions inside. 


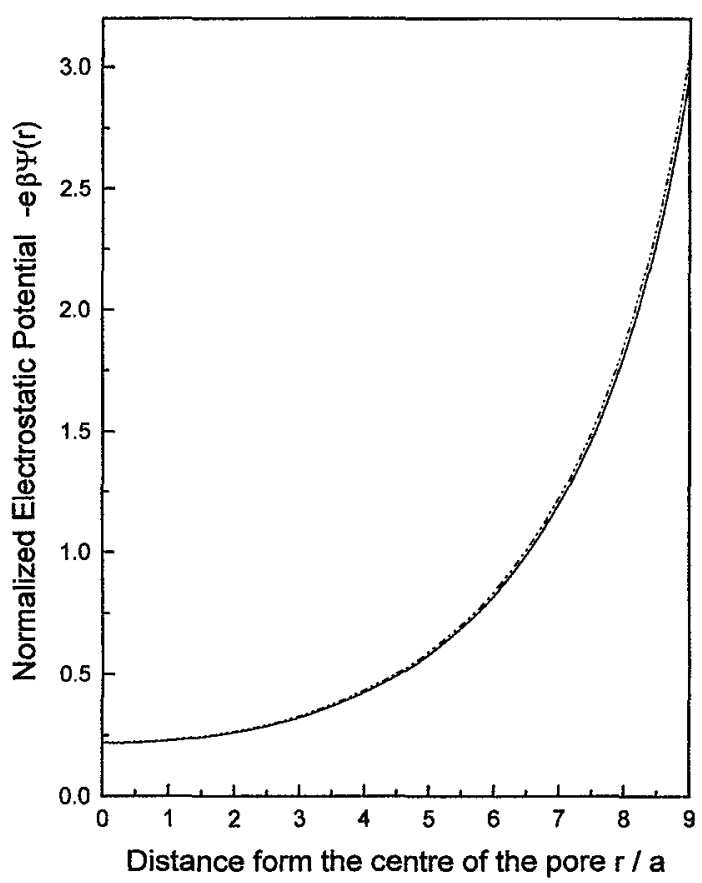

FIG. 2. Potential profile in a pore with a radius $R=9.0 a$. [The solid line is the PB result using Eq. (4) and the dash-dot line is the PB result using Eq. (7).]

$$
\left.\frac{d \psi}{d r}\right|_{r=0}=0 .
$$

At the boundary of the wall, which is defined by the closest approach of the ions $r=R$, the field is determined by the surface charges on the wall at $r=R+a / 2$. For convenience, it is commonly assumed ${ }^{4-7,11}$ that the field is the same at $r=R$ and $r=R+a / 2$ so that

$$
\left.\frac{d \psi}{d r}\right|_{r=R}=\frac{\sigma_{s}}{\epsilon_{0} \epsilon_{r}},
$$

where $\sigma_{s}$ is the surface charge density at $r=R+a / 2$.

Once the potential profile is determined by solving Eq. (1) with the two boundary conditions, the density profile $n_{i}(r)$ of the ions can be calculated from the electrostatic potential since

$$
n_{i}(r)=n_{i 0} \exp \left[-z_{i} e_{0} \psi(r) \beta\right] .
$$

Here, $n_{i 0}$ is the number density of species $i$ in the absence of an external field. From the density profile, we can integrate over the volume of the pore of length $H$ to get the net charge of the electrolyte inside the pore

Net Charge $=2 \pi H \int_{0}^{R}\left[z_{+} e n_{+}(r)-z_{-} e n_{-}(r)\right] r d r$.

\section{TEST OF ELECTRONEUTRALITY}

Calculations are performed for a 1:1 electrolyte inside a pore with a surface charge density $\sigma_{s}=-0.07123 \mathrm{C} \mathrm{m}^{-2}$. The hard sphere diameter $a$, is $0.425 \mathrm{~nm}$. The temperature is

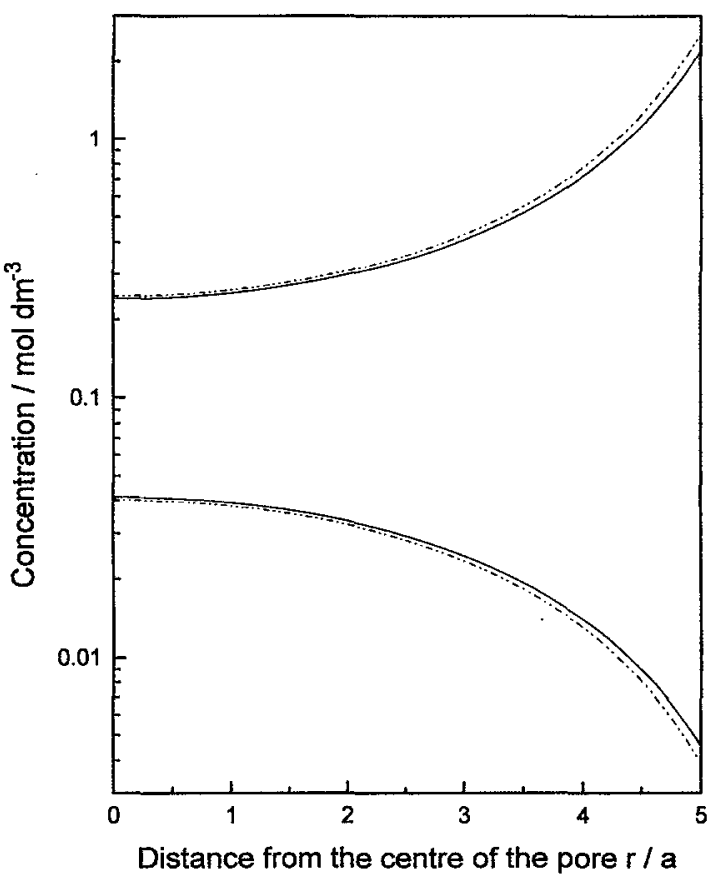

FIG. 3. Density profiles for $R=5.0 a$. [The solid line is the PB result using Eq. (4) and the dash-dot line is the PB result using Eq. (7).]

$298.15 \mathrm{~K}$ and two pore sizes $R=5.0 a, 9.0 a$ are used. The PB equation (1) with the boundary conditions (3) and (4) are solved numerically by a successive over-relaxation method ${ }^{13}$ with several different grid sizes $\Delta r$. As opposed to the shooting method used in Refs. $4-7$, this method does not require any assumption of $\psi$ at the boundaries. The absolute potential is directly obtained. The potential profile calculated for $R=9.0 a$ is shown in Fig. 2. With the calculated potential profile, the density profiles are calculated in concentration units and are shown in Fig. 3 for $R=5 a$ and in Fig. 4 for $R=9 a$. The numerical integration of the density profile using Eq. (6) is performed using the Simpson's method ${ }^{14}$ with different step sizes $\Delta r$ so that extrapolation to zero step size can be obtained. The step sizes in the numerical integration are the same as the grid sizes in the over-relaxation method. Table I gives the results of the integration using different step sizes $\Delta r$. The extrapolation to zero step size is shown in Fig. 5. The results show that the charge inside the pore is less than the charge on the wall. This violation of electroneutrality is more severe for the smaller pore.

It is clear that the boundary condition of Eq. (4) is incorrect and leads to the violation of electroneutrality. Applying Gauss's law to the annular space between $r=R$ and $r=R$ $+a / 2$, the field at $R$ should be higher than that at $R+a / 2$. The proper boundary condition should be

$$
\left.\frac{d \psi}{d r}\right|_{r=R}=\frac{\sigma_{s}}{\epsilon_{0} \epsilon_{r}}\left(\frac{R+a / 2}{R}\right) .
$$

With this new boundary condition, the PB equation is solved again with the same numerical procedures. The potential profile thus obtained is compared with the previous profile in 


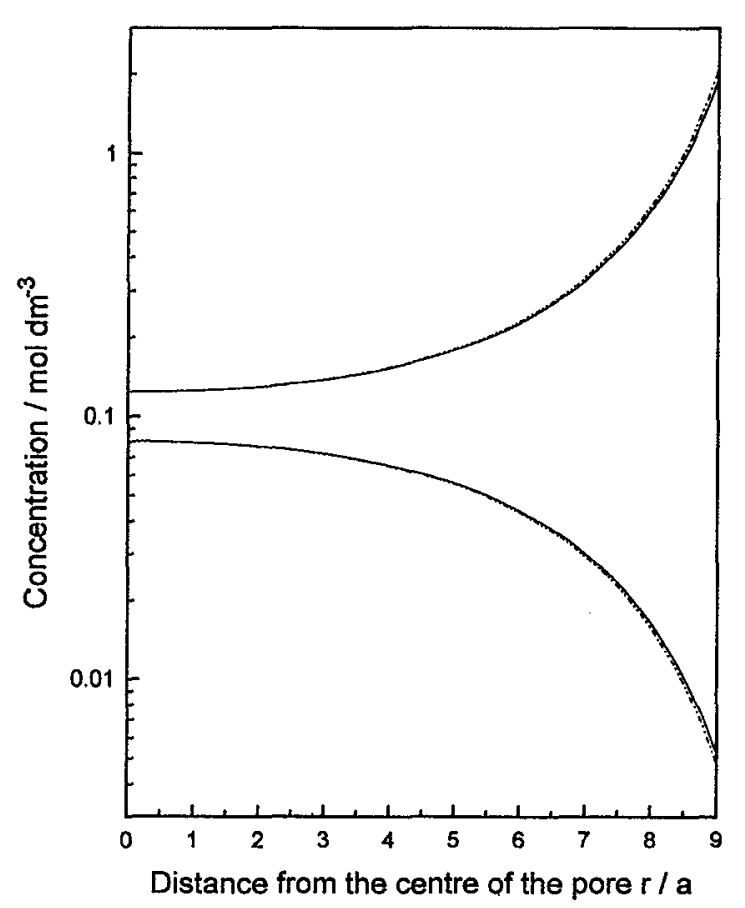

FIG. 4. Density profiles for $R=9.0 a$. (The notation is the same as in Fig. 5.)

Fig. 2 and the corresponding density profiles are shown in Fig. 3. The potential profile is almost identical to that obtained using Eq. (4). The density profiles show some deviations from those obtained using $\mathrm{Eq}$. (4) in the region close to the wall. The net charge inside the pore is again calculated using Simpson's rule using different integration step sizes. The results are tabulated in Table II and the net charge calculated using different step sizes are plotted in Fig. 5. The extrapolation to zero step size shows that the net charge inside the pore now balances the wall charge.

At a closer examination of Table I, the charge imbalance can be quantified by the error in boundary condition (4). According to Gauss's law, the charge inside the cylinder

TABLE I. Net charge inside the pore calculated from the PoissonBoltzmann equation with boundary condition (4).

\begin{tabular}{|c|c|c|c|c|}
\hline$\Delta r / R$ & $\left\langle c_{-}\right\rangle / M$ & $\left\langle c_{+}\right\rangle / M$ & $\begin{array}{c}\text { Excess } \\
\text { number } \\
\text { of counterions } \\
\text { in solution }\end{array}$ & $\begin{array}{c}\text { Net charge } \\
\text { in solution } \\
\text { per wall } \\
\text { area/ } \mathrm{C} \mathrm{m}^{-2}\end{array}$ \\
\hline \multicolumn{5}{|l|}{$a, R=5.0 a$} \\
\hline 0.0002 & 0.02036 & 0.7115 & 125.46 & 0.06441 \\
\hline 0.001 & 0.02026 & 0.7108 & 125.36 & 0.06435 \\
\hline 0.002 & 0.02023 & 0.6986 & 123.15 & 0.06322 \\
\hline Extrap. to 0 & & & & 0.0643 \\
\hline \multicolumn{5}{|l|}{ b, $R=9.0 d$} \\
\hline 0.0002 & 0.04036 & 0.4248 & 407.04 & 0.06720 \\
\hline 0.001 & 0.04034 & 0.4227 & 404.80 & 0.06683 \\
\hline 0.002 & 0.04031 & 0.4126 & 394.10 & 0.06506 \\
\hline Extrap. to 0 & & & & 0.0672 \\
\hline
\end{tabular}

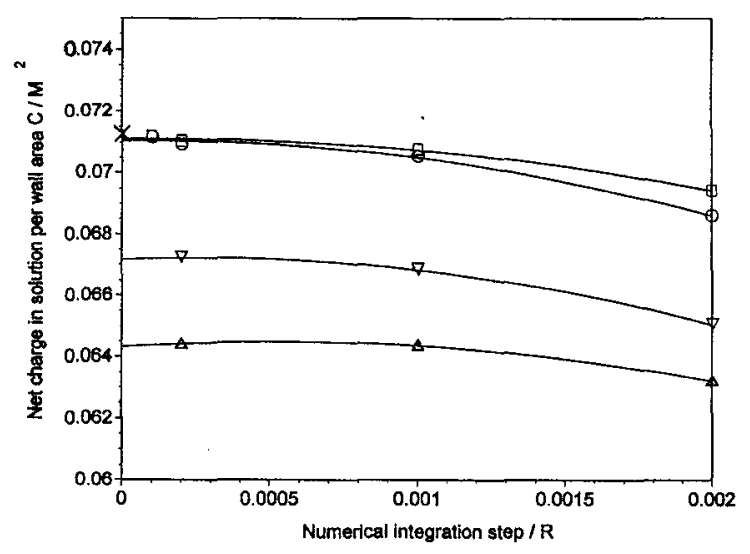

FIG. 5. Net charge of the solution per wall area with different step sizes of integration of the Poisson-Boltzmann equation. [The inverted triangles are the case of $R=5.0 a$ and $R=9.0 a$, respectively, using boundary condition (4). The squares and circles are the case of $R=5.0 a$ and $R=9.0 a$, respectively, using boundary condition (7). The cross is the surface charge density on the wall.]

should be equal to the surface area times the electric field normal to the surface. The amount of missing charge leading to non-neutrality is therefore $2 \pi H(a / 2) \sigma_{s}$. The percentage of charge imbalance is $(a / 2) /(R+a / 2) \times 100 \%$.

\section{CONCLUSIONS}

The Poisson-Boltzmann model is a convenient tool of studying ions in charged capillaries and the solution can be readily achieved by an over-relaxation method without the assumption of a reference potential. The boundary condition at the pore wall, however, should be carefully set up to avoid a violation of electroneutrality. Several references in the literature have chosen the incorrect boundary condition and which violates the electroneutrality condition.

TABLE II. Net charge inside the pore calculated from the PoissonBoltzmann equation with boundary condition (5).

\begin{tabular}{lllcl}
\hline \hline & $\left\langle c_{-}\right\rangle / M$ & $\left\langle c_{+}\right\rangle / M$ & $\begin{array}{c}\text { Excess } \\
\text { number } \\
\text { of counterions } \\
\text { in solution }\end{array}$ & $\begin{array}{c}\text { Net charge } \\
\text { in solution } \\
\text { per wall } \\
\text { area/C } \mathrm{m}^{-2}\end{array}$ \\
\hline $\mathrm{a}, R=5.0 a$ & & & & \\
0.0001 & 0.01929 & 0.7831 & 138.66 & 0.07118 \\
0.0002 & 0.01929 & 0.7816 & 138.38 & 0.07104 \\
0.001 & 0.01928 & 0.7786 & 137.85 & 0.07076 \\
0.002 & 0.01926 & 0.7644 & 135.27 & 0.06944 \\
Extrap. to 0 & & & & 0.0712 \\
& & & & \\
$\mathrm{~b}, R=9.0 a$ & & & 430.92 & 0.07115 \\
0.0001 & 0.03971 & 0.4467 & 429.60 & 0.07093 \\
0.0002 & 0.03971 & 0.4455 & 427.14 & 0.07052 \\
0.001 & 0.03970 & 0.4431 & 415.46 & 0.06860 \\
0.002 & 0.03967 & 0.4321 & & 0.0712 \\
Extrap. to 0 & & & & \\
\hline \hline
\end{tabular}




\section{ACKNOWLEDGMENTS}

The authors acknowledge the Conference and Research Grants Committee of The University of Hong Kong and the Research Grants Council for the financial support of this work.

${ }^{1}$ S. Levine, J. R. Marriot, G. Neale, and N. Epstein, J. Colloid Interface Sci. 52, 136 (1975).

${ }^{2}$ W. Olivares, T. L. Croxton, and D. A. McQuarrie, J. Phys. Chem. 84, 867 (1980).

${ }^{3}$ V. L. Sigal and Y. Y. Ginsburg, J. Phys. Chem. 85, 3730 (1981).

${ }^{4}$ V. Vlachy and A. D. J. Haymet, J. Am. Chem. Soc. 111, 477 (1989).
${ }^{5}$ V. Vlachy and A. D. J. Haymet, J. Electroanal. Chem. 283, 77 (1990).

${ }^{6}$ V. Vlachy and A. D. J. Haymet, Aust. J. Chem. 43, 1961 (1990).

${ }^{7}$ B. Jamnik and V. Vlachy, J. Am. Chem. Soc. 115, 660 (1993).

${ }^{8}$ M. Lozada-Cassou, J. Phys. Chem. 87, 3729 (1983).

${ }^{9}$ E. Gonzalez-Tovar, M. Lozada-Cassou, and D. Henderson, J. Chem. Phys. 83, 361 (1985).

${ }^{10}$ V. Vlachy and D. A. McQuarrie, J. Chem. Phys. 83, 1927 (1985).

${ }^{11}$ V. Vlachy and D. A. McQuarrie, J. Phys. Chem. 90, 3248 (1986).

${ }^{12}$ L. Yeomans, S. E. Feller, E. Sanchez, and M. Lozada-Cassou, J. Chem. Phys. 98, 1436 (1993).

${ }^{13}$ W. H. Press, B. P. Flannery, S. A. Teukolsky, and W. T. Vettering, Numerical Recipes (Cambridge University, New York, 1986), p. 588.

${ }^{14} \mathrm{~K}$. E. Atkinson, An Introduction to Numerical Analysis, 2nd ed. (Wiley, Singapore, 1989), p. 256. 